\title{
Acquisition of barpressing in rats following experience with response-independent food
}

\author{
KIMBAL L. WHEATLEY \\ Weber State College, Ogden, Utah 84408 \\ ROBERT L. WELKER \\ Mount Holyoke College, South Hadley, Massachusetts 01075 \\ and \\ RAYMOND C. MILES \\ University of Colorado, Boulder, Colorado 80302
}

\begin{abstract}
In a three-group experiment, one group of rats (response-contingent) learned to contact a food cup for Noyes food pellets delivered according to a fixed-ratio schedule of reinforcement. Subjects in another group received a response-independent pellet each time its yoked counterpart earned one. A third (control) group received the same amount of food as the other two groups each day, but the pellets were delivered in mass. Following this training, the rats were placed in a novel experimental chamber in which all responses on a bar were reinforced with the presentation of food pellets. The results showed the response-independent animals to be slower in acquiring the barpress response than the naive control subjects, and the response-contingent subjects to be the fastest.
\end{abstract}

It has been repeatedly demonstrated that administering response-independent electric shocks to dogs and rats may interfere with their subsequent learning to escape 'rom shock when compared with the learning of subjects receiving either prior exposure to escapable shocks or no shocks (e.g., Maier, 1970; Maier, Albin, \& Testa, 1973; Maier \& Testa, 1975; Overmier \& Seligman, 1967; Seligman \& Beagley, 1975; Seligman \& Maier, 1967; Seligman, Rosellini, \& Kozak, 1975). Interference with learning to escape from shock under these conditions has been referred to as "learned helplessness," an interpretation which proposes that subjects learn that their behavior has no effect regarding the presentation and removal of shocks during exposure to responseindependent shocks. This learning of independence is assumed to be the source of interference with the subsequent learning to escape from shock (Maier \& Seligman, 1976; Maier, Seligman, \& Solomon, 1969; Seligman, Maier \& Solomon, 1971).

Welker (1976) reported the results of two experiments that may represent an appetitive parallel to those

This research was supported in part by an NIH predoctoral fellowship awarded to Robert L. Welker, and preparation of the manuscript was aided by NIMH Grant 1 RO3 MH28552-01, awarded to Robert L. Welker, and Weber State College RPGC Grant 32-98951-4053, awarded to Kimbal L. Wheatley. Requests for reprints should be addressed to Robert L. Welker, Department of Psychology, Mount Holyoke College, South Hadley, Massachusetts 01075 , or to Kimbal L. Wheatley, Department of Psychology, Weber State College, Ogden, Utah 84408. interpreted as learned helplessness. In the first experiment, three groups of pigeons were subjected to different preliminary training conditions. Subjects in a response-contingent group received approximately 900 grain presentations over 15 experimental sessions, administered according to a variable-interval (VI) $45-\mathrm{sec}$ schedule, with each grain presentation contingent upon pecking an unilluminated response key. Subjects in a response-independent group received 15 sessions in which grain was delivered but no specific response was required. Subjects in a control group were merely trained to approach and eat from the grain hopper during a single experimental session. Following these pretraining conditions, a foot-treadle was inserted along the chamber wall opposite the response key and each grain presentation was contingent upon a single treadle press (continuous schedule of reinforcement, CRF). Subjects in the response-independent group were slower in acquiring the treadle-press response than subjects in either the response-contingent or control group. The rationale for Welker's second experiment followed from previous experiments on learned helplessness with rats as subjects. In the second experiment, it was determined that interference with the acquisition of the treadle-press response following exposure to response-independent grain deliveries could be enhanced by increasing the response requirement for each grain delivery to fixed ratio (FR) 3. Maier et al. (1973) reported that exposure to response-independent shock produced no interference with acquisition of a simple two-way shuttle response to escape from shock, whereas 
requiring subjects to emit two responses to terminate shock (i.e., leave and return to the side of the shuttlebox in which shock was first encountered) produced sub. stantial interference following response-independent shock. Seligman and Beagley (1975) reported similar data using CRF vs. FR3 schedules for barpress escape responses. Welker concluded that the similarities between his data and those interpreted as learned help. lessness might be viewed as "support for the proposition that learned helplessness may be a phenomenon amenable to investigation with appetitive procedures" (p. 402; see also Maier \& Seligman, 1976).

Several parameters of the Welker (1976) experiment differ from the typical experiment used to investigate learned helplessness. These parametric differences must be resolved in order to futher ascertain the functional similarities between interference with learning in the appetitive and aversive tasks following exposure to response-independent food and shock. First, in the learned helplessness studies, the preliminary shocks are usually administered in an experimental context quite different from the chamber in which escape learning is observed, whereas in the Welker (1976) study, preliminary training and testing were conducted in the same chamber. This is an important consideration in light of recent demonstrations of contextual control over appetitive responding in pigeons (Welker, Tomie, Davitt, $\&$ Thomas, 1974) and the involvement of contextual stimuli in transfer of training (Tomie, 1976a, 1976b). Secondly, a yoked control procedure was not employed in the Welker (1976) experiment. Thus the distribution of grain deliveries may have been dissimilar during preliminary training for subjects in the responsecontingent and response-independent groups. Thirdly, the Welker (1976) study employed pigeons as subjects and the authors are aware of no investigation of learned helplessness with avian subjects. The potential for interspecies differences should be eliminated in initial comparisons of similarities in interference with learning following appetitive and aversive response-independent events. Finally, in the Welker (1976) experiments, subjects were given supplemental feedings in their home cages following experimental sessions in order to maintain a weight criterion. The supplemental feedings may be viewed as response-independent, and the supplemental feeding of subjects in all groups might mitigate differences between groups accrued as a function of the response-independent and response-contingent deliveries of food in preliminary training. No obvious parallel to this problem exists in investigation of learned helplessness with aversive stimuli.

The present experiment was designed, in part, to resolve the parametric differences in investigations of interference with learning in appetitive and aversive tasks following exposure to response-independent events. Rats were exposed to response-contingent or response-independent food deliveries during preliminary training in one experimental context and tested for the acquisition of a barpress response which produced food in a different context. A yoked control procedure was employed for pellet deliveries in the response-contingent and response-independent conditions, and the design precluded the necessity for supplemental feedings during preliminary training. In addition, the experiment was designed to provide information pertinent to an alternative interpretation that has been invoked to account for interference with learning following responseindependent events. This interpretation has been referred to as "response competition," and proposes that interference with learning following responseindependent events is produced via the subjects' acquiring a specific response during exposure to responseindependent events which later competes with the to-be-acquired response. For discussion of several variations of the response competition interpretation, see: Bracewell and Black (1974), Engberg, Hansen, Welker, and Thomas (1972), Glazer and Weiss (1976a, 1976b), Gamzu, Williams, and Schwartz (1973), Maier and Seligman (1976), and Welker, Hansen, Engberg, and Thomas (1973). In the present experiment, the response required during preliminary training for the responsecontingent group was also recorded during the test for acquisition of the new response. It was anticipated that simultaneous recording of both responses would provide information regarding the dynamics of extinguishing one response while acquiring another. This type of information is vital for systematic evaluation of a response competition interpretation.

\section{METHOD}

\section{Subjects}

Twelve Long-Evans hooded male rats raised in a laboratory at the University of Colorado served as subjects. The rats were selected from three litters, weaned at 25 days of age and housed singly in cages in a colony room maintained on a 12/12-h light/dark cycle. The subjects received ad-lib food and water under these conditions until 45 days of age.

\section{Apparatus}

The experimental chambers used in preliminary training consisted of four attached cubicles $(25 \times 25 \times 30 \mathrm{~cm})$ with wire mesh floors and ceilings and flat-black plywood walls. Each cubicle was equipped with a pellet dispenser which delivered $45-\mathrm{mg}$ Noyes pellets to a food cup located $3 \mathrm{~cm}$ from the floor and $3 \mathrm{~cm}$ to the left of center of one of the walls. Contacts with the food cup were recorded by lickometer circuitry in which the rat completed a circuit between the wire mesh floor and food cup. A water spout was located $3 \mathrm{~cm}$ from the floor and $10 \mathrm{~cm}$ to the right of the food cup. The room in which the cubicles were housed was dimly illuminated, and white noise was presented continuously to mask extraneous noise. Experimental variables were manipulated automatically via standard relay circuitry located in a separate room.

The testing chambers were two $30 \times 22 \times 35$ cubicles constructed of transparent and white Plexiglas with grid floors. Each chamber contained a contact-sensitive food cup identical to the ones used in the cubicles during preliminary training. The food cups were located at the center of one of the walls, $3 \mathrm{~cm}$ above the floor, and a Lehigh Valley response lever was located $11 \mathrm{~cm}$ to the left of the food cup and $6 \mathrm{~cm}$ above the 
tloor. The chambers were housed in separate sound-attenuating hulls with white plastic interiors illuminated by $40-\mathrm{W}$ bulbs. Ventilation fans further masked intraneous noise.

It should be noted that the preliminary-training cubicles Uitfered from the testing chambers on several dimensions (e.g., ambient luminance, ambient noise. texture of floor and walls, size. etc.). The two experimental contexts were similar with respect to the food cups, delivery of $45 \mathrm{-mg}$ Noyes pellets. and the sound accompanying activation of the pellet dispenser.

\section{Procedure}

The experimental design employed four triads of subjects, with each triad selected from a common litter. When the rats "lire 45 days of age, they were placed in the preliminary training aubicles and remained there for $24 \mathrm{~h}$ a day for a total of 15 days. One subject from each triad was assigned to a response-contingent group and received its pellets by contacting the foud cup. The schedule for pellet delivery was CRF during the first day, FRS during the second day, and I R 25 during Days 3-15. No shaping procedures were used in acquisition of the food-cup-contacting response. The second member of each triad was assigned to a response-independent group, and its pellet deliveries were loked to those of a subject in the response-contingent group. In other words. the response-independent subject of each triad received a pellet every time the response-contingent subject from the same triad earned one. The third subject within each triad was designated a control and received the same number of pellets as its respective response-contingent and responseindependent counterparts. but the pellets were delivered in mass in the food cup at regular $24-\mathrm{h}$ intervals $(3 \mathrm{p.m}$.). The pellet dispenser was not activated during these feedings. The vertically oriented plastic tubing connecting the food cup and pellet dispenser was disconnected and the appropriate number of pellets were funneled in mass into the tube. Immediately prior to the massed feeding for the 15 th day of preliminary training, subjects in the control group were trained to approach the tood cup when the pellet dispenser was activated. Pellet deliveries were determined by an experimenter, and the criterion for successful training was approaching the food cup and consuming a pellet immediately after each of 20 successive pellet deliveries.

At the end of the 15 th day of preliminary training, all subjects were returned to single cages in the colony room and deprived of food for $24 \mathrm{~h}$. They were then placed in the novel tusting chambers at a constant time daily. Each pellet delivery in the testing chamber was contingent upon a single barpress (CRF), and subjects were placed in the chambers for $2 \mathrm{~h}$ daily until they had emitted at least 50 barpresses. Barpresses and contacts with the food cup were recorded simultaneously in iuccessive 2 -min intervals during testing sessions. When subjects tailed to barpress, or emitted only a few barpresses during a resting session, supplemental feedings were given in order to maintain them at no less than $85 \%$ of their weights at the end of the 15 th day of preliminary training. Supplemental feedings were given approximately $2 \mathrm{~h}$ after the completion of a testing session. Each supplemental feeding consisted of placing the subjects in the cubicles used for preliminary training and feeding them in accordance with the conditions they experienced in preliminary training: response-contingent, response-independent, or massed pellet deliveries. An FR25 schedule was used for subjects in the response-contingent group with pellet deliveries for subjects in the response-independent group yoked to those of a subject in a response-contingent group. Subjects in the control group received an equivalent number of pellets in mass. This supplemental feeding procedure was continued even after a given subject in a response-contingent group obtained the criterion for acquisition of barpressing. Upon reaching a criterion, a subject from the response-contingent group was no longer placed in the testing chamber, but instead was placed in the cubicle used for preliminary training at the time that a response- independent subject from the same triad was to be given supplemental feeding (i.e., following the testing session for the response-independent subject).

\section{RESULTS}

The frequency of contacting the food cup during preliminary training differed substantially among the three groups of subjects. The means for this measure on the 15 th day of preliminary training were $5,025.3$, 498.7 , and 416.2 for subjects in the response-contingent, response-independent, and control groups, respectively. Thus, during the 15 th day of preliminary training, the mean number of pellets consumed per rat was roughly 200. Approximately $15 \mathrm{~min}$ were required to train each rat in the control group to approach the food cup upon activation of the pellet dispenser during the 15 th day of preliminary training. The number of pellets delivered for this training ranged from 35 to 52 .

The three groups did not differ with regard to the average (mean or median) time taken to emit the first barpress in the testing chamber (medians: $3 \mathrm{~min}$, response-contingent group; $2 \mathrm{~min}$, response-independent group; $2 \mathrm{~min}$, control group). Individual scores for the time elapsing between the 1 st and 50th barpresses are presented in Table 1. Notice that in each triad the response-contingent subject emitted 50 barpresses in less time than that taken for the respective control subject, and in three of the four triads, the response-independent subjects required more time for this measure than their respective controls. When considering either means or medians for this measure, the three groups of subjects may be rank-ordered regarding speed of acquiring the barpress as follows: response-contingent, control, and response-independent. The results of a Friedman test for matched subjects indicated that the differences among the groups were reliable $(\mathrm{p}<.05)$.

In order to analyze the time course for acquisition of barpressing, barpresses were tabulated in Vincentized form. Figure 1 presents barpress acquisition data for each group in terms of the mean time required to complete the first block of 10 responses, the second block of 10 responses, etc. The mean time to complete 10 barpresses was transformed by taking the square root of the time required for each subject. Computation

Table 1

Number of Minutes Elapsing Between the 1st and the 50th Barpresses for Individual Subjects

\begin{tabular}{cccc}
\hline Triads & $\begin{array}{c}\text { Response } \\
\text { Contingent }\end{array}$ & $\begin{array}{c}\text { Response } \\
\text { Independent }\end{array}$ & Control \\
\hline 1 & 24 & 40 & 28 \\
2 & 166 & 224 & 264 \\
3 & 32 & 916 & 86 \\
4 & 62 & 1022 & 158 \\
Mean & 71 & 550.5 & 134 \\
Median & 47 & 570 & 122 \\
\hline
\end{tabular}




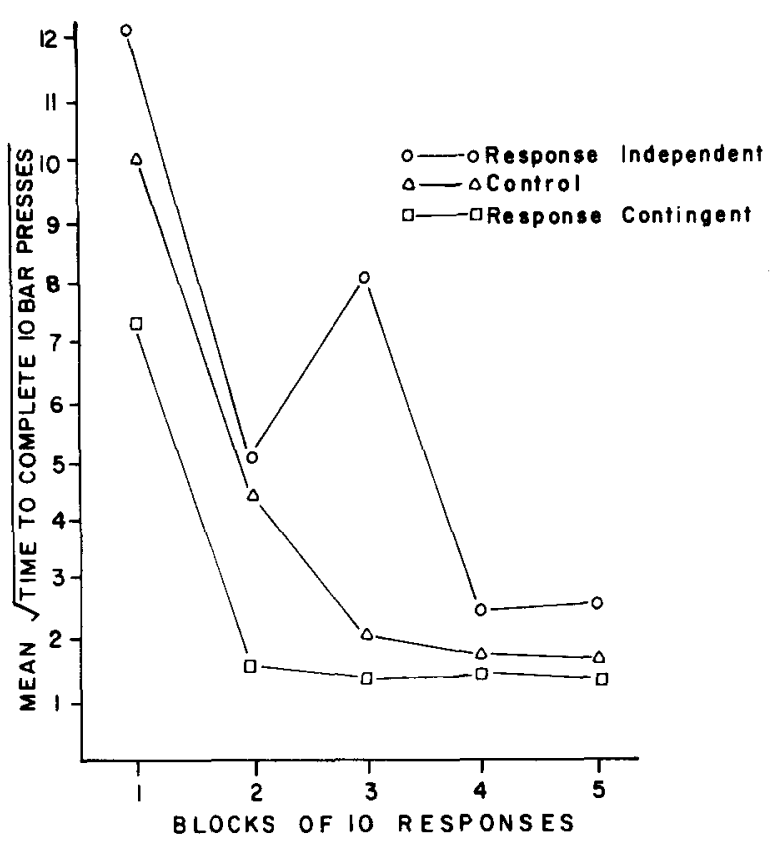

Figure 1. Mean transformed data for each group indicating the time required to emit successive blocks of 10 responses.

of the $F_{\max }$ statistic indicated that the square root transformation homogenized the variance, allowing analysis of variance procedures. A mixed analysis of variance (treating the groups as independent) yielded a marginally reliable main effect of groups, $F(2,9)=3.91$, $.05<\mathrm{p}<.10$, and a reliable main effect of blocks, $F(4,36)=8.78, p<.001$, but no interaction, $F<1$. Newman-Keuls comparisons regarding the groups indicated that the response-contingent subjects completed the 50 barpresses faster than the controls which were in turn faster than the response-independent subjects (all post hoc comparisons were computed at .01 alpha levels).

Figure 2 depicts changes in the rate of contacting the food cup during successive stages in the acquisition of barpressing for the three groups. Contacts with the food cup were totaled for each subject during the time taken to emit successive blocks of 10 barpresses. These totals were converted to rates (contacts/number of minutes required for 10 barpresses), and the mean rate of contacting the food cup was then determined for the three groups for each block. As can be seen in the figure, the mean rate of contacting the food cup increased for all three groups over successive 10 response blocks. A mixed analysis of variance indicated a reliable effect of blocks, $F(4,36)=12.76, p<.001$. The subjects from the three groups differed in the rate at which they increased contact with the food cup across the response blocks $F(8,36)=14.89, p<.001$, but the main effect of groups did not achieve statistical reliability, $F(2,9)=$ $2.74, \mathrm{p}>.10$.

Figure 3 displays the acquisition of barpressing in conjunction with contacting the food cup for a responsecontingent and response-independent pair selected from one of the triads. Data points represent response rates computed for successive 2 -min periods, where the absence of a point for any given 2 -min period indicates no responses for that interval. Notice that the rate of contacting the food cup was sporadic across time and that it was generally higher for the response-contingent subject than for the response-independent subject. The rate of contacting the food cup increased immediately prior to the emergence of an acquisition curve for barpressing for both subjects, and, as the barpressing rates began to stabilize, the rate of contacting the food cup decelerated. This transient increase in rate of contacting the food cup at the point of emergence of an acquisition curve for barpressing was observed in the individual records of all of the 12 subjects, and is obscured in the group summaries presented in Figure 2.

\section{DISCUSSION}

The elevated frequency of contacting the food cup during preliminary training for subjects in the responsecontingent group relative to that of subjects in the response-independent and control groups indicates that the FR25 contingency was effectively controlling the behavior of contacting the food cup.

Interference with the acquisition of barpressing for subjects in the response-independent group relative to subjects in both the response-contingent and control groups is consistent with the data reported by Welker (1976). More specifically, in both instances the interference was evident in the time elapsing between the 1 st and 50th response and no differences were observed among the groups in the time required to emit the first response. The latter finding indicates that the interference is not easily attributed to differences in general

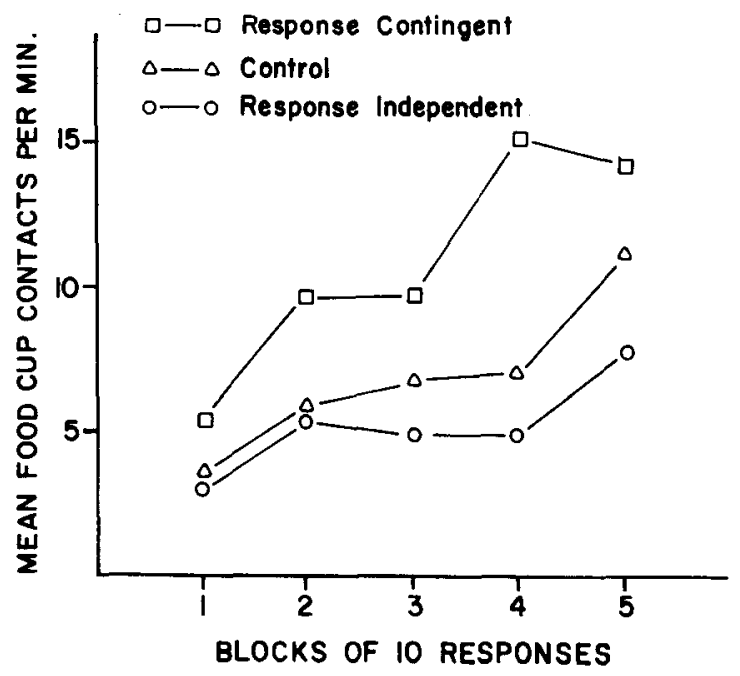

Figure 2. Group means for the number of times the subjects contacted the food cup over the five 10-response blocks. 


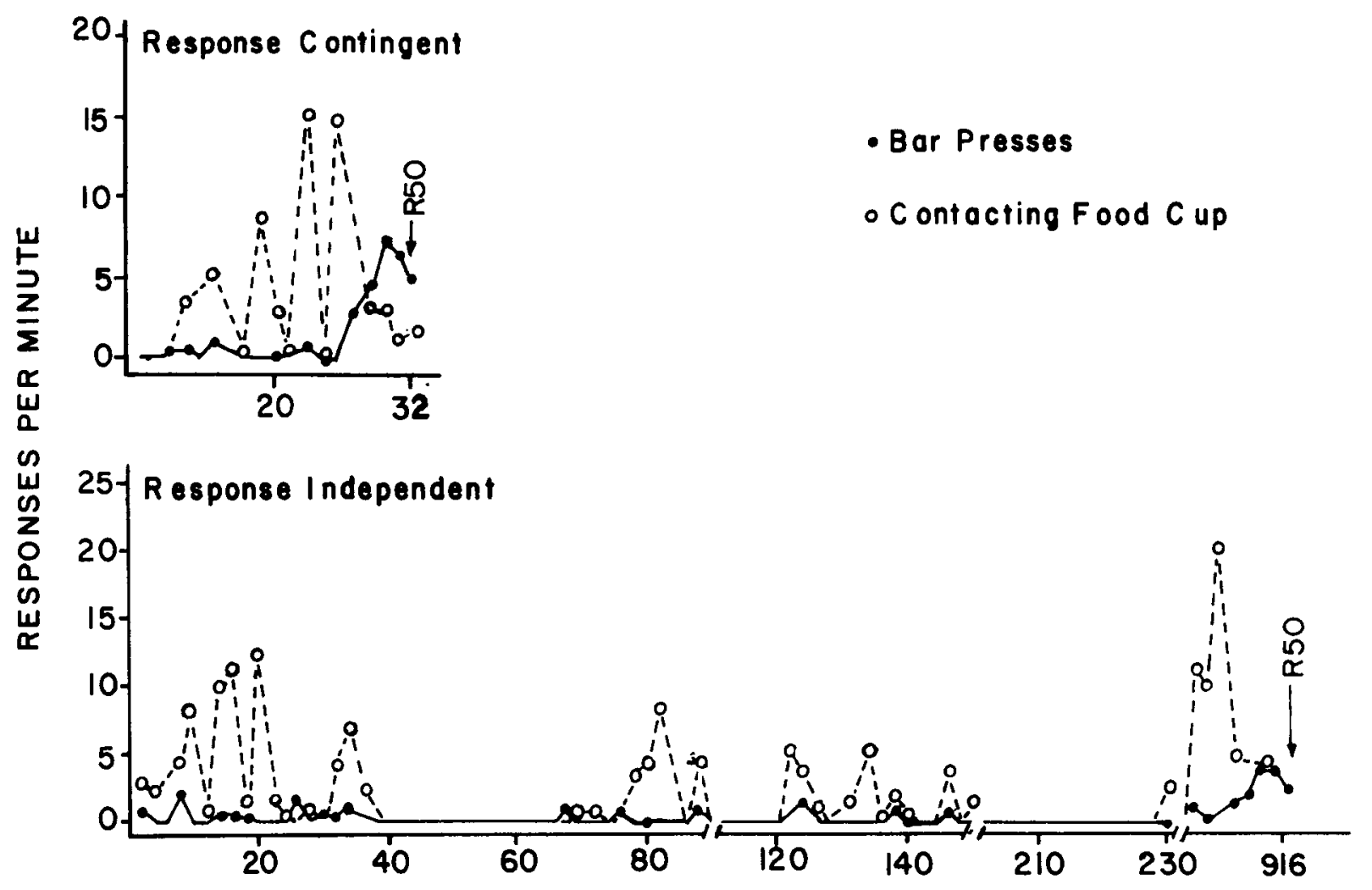

\section{TIME IN MINUTE'S TO 50 th B AR PRESS}

Figure 3. Acquisition curves of barpressing in conjunction with rates of touching the food cup for a response-contingent and response-independent subject from a yoked pair. See text for explanation.

activity levels among the groups. The multiple parametric differences between this experiment and Welker's (1976) extend the generality of the conditions under which interference with the acquisition of appetitive responses may be observed following exposure to response-independent food, and strengthen the proposed similarities between this phenomenon and interference with learning to escape from shock following exposure to response-independent shock (cf. Maier \& Seligman, 1976; Welker, 1976).

The results of this experiment and those of Welker (1976) appear to differ with the results of the typical learned-helplessness experiment, however, in the degree of permanence of interference with learning (e.g., Maier et al., 1973; Seligman et al., 1975). It might be argued that learned helplessness is reflected by failure to learn to escape from shock, whereas the present data and those of Welker (1976) indicate only a transient interference with learning. It must be noted that observations of failures to learn are heavily dependent upon the duration of exposure to a learning task. Most experiments designed to study learned helplessness employ single testing sessions, whereas the present experiment employed protracted testing sessions. Had we chosen to limit our 2-h testing sessions to a total of five, two subjects in the response-independent group (from Triads 3 and 4) would have reflected a failure to learn to barpress. For additional discussion of the variables affecting apparent differences in the degree of permanence of interference with learning to procure food or escape from shock following exposure to response-independent food and shock, see Welker (1976).

According to a learned-helplessness interpretation of the present data, the subjects in the response-independent group were slowest in acquiring barpressing because they learned about the independence of pellet deliveries and their behavior during preliminary training. This learning could possibly result in development of a concept that "Nothing I do matters" with regard to this particular event (Maier et al., 1969). Assuming that the interference with acquisition of barpressing for subjects in the response-independent group was mediated via their development of such a concept in reference to food delivery, it is not immediately obvious why the subjects in the control group failed to exhibit the same degree of interference. Their massed food deliveries were also administered in a manner that was independent of their behavior. Pursuing this line of reasoning to an extreme, one would expect that most animals used in behavioral experimentation requiring food deprivation would be to some degree helpless. This would be so because food deprivation usually involves feeding 
subjects at a particular time of day in a manner that is independent of their behavior. Animals that are fed in accordance with this type of procedure, however, acquire a variety of responses for food with relatively little difficulty. The crucial difference between food deliveries for the response-independent and control groups was the temporal distribution of pellet deliveries. Pellets were delivered in bulk at the same time daily to the control group, whereas the response-independent group received single pellets distributed over a wider range of time. Although exact records of this distribution were not obtained, animals exposed to conditions similar to those of preliminary training for the responsecontingent group eat about 20 meals of approximately 20-min duration in $24 \mathrm{~h}$, with successive meals separated by approximately $60 \mathrm{~min}$ of fasting (Collier, Hirsch, \& Hamlin, 1972). It is most probable that differences in the temporal distribution of food pellets are responsible for the slower acquisition of barpressing by subjects in the response-independent group than the control group.

This observation and inference may prove useful in specifying the mechanisms involved in learning about the independence between an environmental event and behavior. It has been proposed that such learning is mediated by the subject's being sensitive to the conjoint probabilities of the event occurring in the presence and absence of a particular behavior (Maier et al., 1969; Maier \& Seligman, 1976; Seligman et al., 1971). When environmental events are administered in a responseindependent manner, the probability that the event will occur in the presence of a particular behavior is assumed to be equal to the probability of the event occurring in the absence of that behavior. Given this framework, it is relatively easy to visualize how an animal might develop the concept of independence between a particular behavior and a particular environmental event. The animal must experience the event about equally often in the presence and absence of a given behavior. It is more difficult to visualize how an animal might discover that none of its behaviors are related to the event. In order for this to occur, the subject must surmise that the probability of an event's occurrence is equal given the presence and absence of a variety of behaviors. In other words, the organism must be actively engaged in many different behaviors during successive occurrences of the event in order to learn that none of them affect the event. It has been suggested that temporal contiguity between a variety of behaviors and the occurrence of an event might lead to the development of the concept that everything I do matters, "learned omnipotence" (Rachlin, 1976). The shortcomings of this position are evident only if one considers that an organism experiencing an event paired with one behavior must realize that the event is occurring in the absence of other behaviors that have been paired with the event in the past. This consideration challenges temporal contiguity as a sufficient condition for learning, and may perhaps be visualized more readily from another perspective.

Baum (1973) and others (cf. Rachlin, 1976; Staddon \& Simmelhag, 1971) distinguish between the role of contiguity and correlation in the control of behavior by instrumental contingencies. When an animal is exposed to a contingency (e.g., CRF schedule of positive reinforcement), it experiences contiguity between its behavior and the reinforcing event, and seems to learn that the probability of occurrence of the event is greater in the presence of a particular behavior than in the absence of the behavior (i.e., a correlation between the rate of response and probability of occurrence of the event). The organism may control to some degree, dependent upon the schedule of reinforcement, the probability of occurrence of the event by increasing or decreasing the frequency with which it emits the behavior. In instances in which events are delivered independently of the organism's behavior, the organism will most likely learn about the independence only if two conditions are realized: (1) it experiences the event paired with a variety of behaviors, and (2) there is no opportunity for an adventitious correlation to exist between the rate of occurrence of any particular behavior or set of behaviors and the rate of occurrence of the event. These conditions are most probably fulfilled when the temporal distribution of the responseindependent event is highly variable and lengthy in tervals occur between some of the successive presentations of the event.

Skinner's (1948) classic demonstration of the emergence of stereotyped behaviors during the delivery of response-independent food to pigeons has often been used as a basis for opposition to the proposal that nonhuman animals may learn about the independence of an event and their behavior. It is of interest that the few convincing demonstrations of "superstitious" behavior in animals (cf. Skinner, 1948; Staddon \& Simmelhag, 1971) employed conditions which fostered adventitious correlations between the rate of a particular behavior, once emerged, and the probability of delivery of food (i.e., short intervals between successive food deliveries and restricted variability for these intervals). These conditions generate a scalloping pattern of the superstitious behavior which results in a greater probability of the delivery of food in the presence of that behavior than in its absence. Skinner (1948) and Staddon and Ayres (1975) both noted that increasing the interval between response-independent food deliveries reduces the probability that food delivery will occur in the presence of the superstitious behavior and may even preclude the emergence of the latter.

Given that temporal regularity and short interevent intervals are necessary for the emergence of "superstitious" behaviors, it is improbable that the retarded acquisition of barpressing by subjects in the responseindependent group of the present experiment may be 
attributed to competition from a "superstitious" behavior conditioned during preliminary training. The simultaneous recordings of barpressing and contacting the food cup also reduce the applicability of a responsecompetition interpretation. It was reasoned on an a priori basis that competition might occur between contacting the food cup and barpressing because the subjects could not press the bar and contact the food cup at the same time. Subjects in the response-contingent group, however, acquired barpressing the fastest, even though they contacted the food cup at a higher rate during the acquisition of barpressing than subjects in either the response-independent or control group. It might be argued that these data refute the notion that barpressing and contacting the food cup were incompatible behaviors, and that the inverse was true (i.e., they were facilitory). An argument such as this suggests that a priori predictions regarding response compatibility relationships are impossible. The argument is also rendered tenuous by its implication that response competition occurs only in the response-independent group and involves unspecified "superstitious" behaviors, while not occurring in the response-contingent group, which was pretrained to emit a behavior that could logically be expected to compete with barpressing.

The rapid acquisition of barpressing by subjects in the response-contingent group could be interpreted as evidence that exposure to a response-reinforcer contingency enhances learning about a new contingency via a process that might be considered the complement of learned helplessness (see Eisenberger, Park, \& Frank, 1976; Engberg, Hansen, Welker, \& Thomas, 1972). The rationale regarding the learning of independence developed herein suggests that enhancement of learning about new contingencies would be facilitated with successive changes in the response topography required for each contingency. Each contingency would involve a common element, a correlation between the rate of some behavior and the delivery of food. With mastery of successive contingencies and the requisite change in response topographies, the subject may develop the concept of "something I do matters" regarding a particular reinforcing event.

\section{REFERENCES}

Baum, W. M. The correlation-based law of effect. Journal of the Experimental Analysis of Behavior, 1973, 20, 137-153.

BraCEWELl, R. J., \& BLACK, H. H. The effects of restraint and non-contingent preshock on subsequent escape learning in the rat. Learning and Motivation, 1974, 5, 53-69.

Collier, G., Hirsch, E., \& Hamlin, P. H. The ecological determinants of reinforcement in the rat. Physiology and Behavior, 1972, 9, 705-716.

Eisenberger, R., Park, D. C., \& Frank, M. Learned industriousness and social reinforcement. Journal of Personality and Social Psychology, 1976, 33, 227-232.

Engberg, L. A., Hansen, G., Welker, R. L., \& Thomas, D. R. Acquisition of key-pecking via autoshaping as a function of prior experience: "Learned laziness"? Science, 1972, 178, 1002-1004.

Gamzu, B. R., Williams, D. R., \& Schwartz, B. Pitfalls of organismic concepts: “Learned laziness"? Science, 1973, 171, 367-368.

Glazer, H. I., \& WEIss, J. M. Long-term and transitory interference effects. Journal of Experimental Psychology: Animal Behavior Processes, 1976, 2, 191-201. (a)

Glazer, H. I., \& Weiss, J. M. Long-term interference effect: An alternative to learned helplessness. Joumal of Experimental Psychology: Animal Behavior Processes, 1976, 2, 202-213. (b)

MAIER, S. F. Failure to escape traumatic electric shock: Incompatible skeletal-motor responses or learned helplessness? Learning and Motivation, 1970, 1, 147-169.

Maier, S. F., Albin, R. W., \& Testa, T. J. Failure to learn to escape in rats previously exposed to inescapable shock depends upon the nature of the escape response. Journal of Comparative and Physiological Psychology, 1973, 74, 471-492.

Maier, S. F., \& Seligman, M. E. P. Effects of inescapable shock upon subsequent escape and avoidance responding. Journal of Experimental Psychology: General, 1976, 105, 3-46.

Maier, S. F., Seligman, M. E. P., \& Solomon, R. L. Pavlovian fear conditioning and learned helplessness. In B. A. Campbell \& R. M. Church (Eds.), Punishment and aversive behavior. New York: Appleton-Century-Crofts, 1969.

MaIER, S. F., \& TEsta, T. J. Failure to learn to escape by rats previously exposed to inescapable shock is partly produced by associative interference. Journal of Comparative and Physiological Psychology, 1975, 88, 554-564.

Overmier, J. B., \& Seligman, M. E. P. Effects of inescapable shock upon subsequent escape and avoidance responding. Journal of Comparative and Physiological Psychology, 1967, 63. 27-33.

Rachlin, H. Introduction to modern behaviorism. San Francisco: Freeman, 1976.

Seligman, M. E. P., \& Beagley, G. Learned helplessness in the rat. Journal of Comparative and Physiological Psychology, 1975, 88. 534-541.

Seligman, M. E. P., \& Maier, S. F. Failure to escape traumatic shock. Journal of Experimental Psychology, 1967, 74, 1-9.

Seligman, M. E. P., Maier, S. F., \& Solomon, R. L. Unpredictable and uncontrollable aversive events. In F. R. Brush (Ed.), Aversive conditioning and learning. New York: Academic Press, 1971.

Seligman, M. E. P., Rosellini, R. A., \& Kozac, M. J. Learned helplessness in the rat: Immunization, time course and reversibility. Journal of Comparative and Physiological Psychology, $1975,88,542-547$.

SKINNER, B. F. Superstition in the pigeon. Journal of Experimental Psychology, 1948, 38, 168-172.

Staddon, J. E. R., \& Ayres, S. L. Sequential and temporal properties of behavior induced by a schedule of periodic food delivery. Behavior, 1975, LIV 1-2, 26-49.

Staddon, J. E. R., \& Simmelhag, V. L. The superstition experiment. Psychological Review, 1971, 78, 3-43.

TOMIE, A. Interference with autoshaping by prior contextual conditioning. Journal of Experimental Psychology: Animal Behavior Processes, 1976, 2, 323-334. (a)

TOMiE, A. Retardation of autoshaping: Control by contextual stimuli. Science, 1976, 192, 1244-1246. (b)

WELKER, R. L. Acquisition of a free-operant response in pigeons as a function of prior experience with response-independent food. Learning and Motivation, 1976, 7, 394-405.

Welker, R. L., Hansen, G., Engaerg, L. A., \& Thomas, D. R. Pitfalls of organismic concepts: "Learned helplessness"? Science, 1973, 181, 368-369.

Welker, R. L., Tomie, A., Davitt, G. A., \& Thomas, D. R. Contextual stimulus control over operant responding in pigeons. Journal of Comparative and Physiological Psychology, 1974, 86, $549-562$.

(Received for publication September 23, 1976; revision accepted March 15, 1977.) 